\title{
Lambda z Upper Limit TAU
}

National Cancer Institute

\section{Source}

National Cancer Institute. Lambda z Upper Limit TAU. NCI Thesaurus. Code C147482.

The upper limit on time for values to be included in the calculation of Lambda $z$, calculated within a dosing interval. 